\section{Clinitul apectures}

DEIIVERED AT

\section{CHARING CROSS HOSPITAL.}

BY

HYDE SALTER, M.D., F.R.S.,

FELLOW OF THE ROTAL COLLEGE OF PHYSICIANS ; LECTURER ON PHYSIOLOGY AND PATHOLOGY AT CHARING CROSS HOSPITAL MEDICAL SCHOOL; AND ASSISTANT. PHYSICIAN TO THE HOSPITAL.

Lecture VIII.-On the Diagnosis of Dropsies. (Concluded.)

Case I. General Mechanical Dropsy from Disease of the Heart.-CAse II. General Mechanical Dropsy from Disease of the Lungs. - CasE IIr. Acute Dropsy from Exposure to Cold.-Case Iv. Dropsy after Scarlatina.-CASE v. OEdema of Feet from Pressure of Enlarged Liver.-Case vi. Ascites with Atrophied Liver.-Case vir. Ascites with Enlarged Liver. -The Relation of Ascites to CEdema of the Legs.

Case I. George Gelden, aged 44, was admitted into the hospital for shortness of breath and swelling of the legs. For twenty years he was a waiter at an hotel, and drank to excess; but he has lately been a light porter, and has drunk moderately. For the last few years he has suffered occasionally from gout; and at the end of last year, about six months ago, was taken with a severe cold on his chest. Six weeks ago his legs began to swell, and have rapidly increased in size, the swelling having gradually extended from the ankles up to the thighs. At the time of his admission, the legs were enormously large, hard, tight, and pitting deeply on sustained pressure. There has been no œdema about the face, body, or upper extremities. There is but little cough or expectoration; breath very short on the least exertion, accompanied with palpitation; while perfectly at rest the breathing is tranquil. On listening over the apex of the heart a strong systolic bruit is hecerd. No albumen in the urine.

What was the diagnosis in this case? Cardiac dropsy, due to mitral regurgitation. And on what was this diagnosis based? In the first place, the dropsy had the distribution of "general mechanical" dropsy, commencing at the lowest part of the body, and gradually creeping up; then we had the essential symptom of dyspnoea present; then the freedom of the upper parts of the body from puffiness, and, above all, the absence of albumen in the urine, relieved the kidneys from all onus. The only doubt, then, at this stage of the inquiry, would be, whether the seat of the mechanical impediment giving rise to the dropsy was the heart or lungs, whether it was a case of cardiac or pulmonary dropsy, whether it was due to bronchitis or heart-disease. This doubt was soon solved; for not only was the amout of mischief found in the lungs inadequate to the production of such results, but, on exploring the heart, we found enough to explain everything - a suitable and sufficient cause for all the symptoms-a grave derangement of the heart's machinery, such as would, sooner or later, give rise to all the effects of mechanical blood-stasis. The effects were such as pointed to the cause; and, on searching for the cause, we found physical evidence of its presence. The chain of evidence was then complete.

I may mention one thing in relation to this case, though it has not to do with the question of diagnosis - the special object of this lecture. It is, that it offers a very good example of the wonderful power of hydragrogue catharsis in reducing dropsy of this kind. At the present time, after having been in the hospital three weeks, the swelling of this man's legs is almost gone. Its cause-the heart-disease-is, of course, exactly the same; but we have been pumping out of him five or six watery motions every morning by means of half-drachm doses of compound scammony powder; and this discharge of water has, by reducing the volume of the blood, so disencumbered the heart's damaged machinery, and, at the same time, so stimulated absorption, that the result is what you see.

CAsE II. James Shearer, aged 42, has served for twenty-one years in the Scots Fusilier Guards, and was in the Crimea; otherwise has been on home duty; had rheumatic fever twice, but, with that exception, has always enjoyed good health, and the rheumatism did not affect his heart or chest : no consumption in his family. His illness dates from April twelvemonth. He was walking down to $\mathrm{New}$ Cross with his wife and children, and noticed a pain in his right side along the lower ribs, rising towards the armpit. In a month the pain had risen higher in the side, and was accompanied with a tightness of the chest and a sense of oppression of the breathing. He seemed quite well as long as he was sitting still ; but when he began to move, he lost his breath. He had a slight cough, mostly dry; and what little expectoration he had was chiefly in the morning. The next thing he noticed was a swelling in the lefit ankle, and shortly after in the right; it soon gradually extended up the legs; by November the swelling had reached the top of the thighs. No other part of the body was swollen, except that he felt a little tightness round the belly. He mostly lay orr the left side (the right being the affected one). By this time the cough was very bad, in paroxysms of four or five minutes duration; the spitting more abundant; the sputum grey, containing no blood, not even rusty. In December he spat blood, ten days in succession, five or six times a day ; but the phlegm was free of blood, and the blood of phlegm -the two perfectly distinct. At this time the sputum had a peculiar taste, that it had not had before; but there was no change in its appearance, nor did the patient ever notice any offensive smell with it, nor did any one else. 'T'he spitting of blood never returned but on one occasion, the morning after the illuminations for the Prince of Wales's marriage; he had been out on that occasion, and coughed violently on coming home. From December to April the swelling of the feet and legs got less; the breathing slightly better, but still very bad; the coughing and spitting night and morning, though less than there had been. He went on slightly improving (the breathing becoming clearer, but the swelling of the legs never entirely leaving him) till the end of May, when he was called out for eight days' drill. His breathing was equal to slow marching, but he could not manage the "double", which doubled him up; he also drank too freely. The result was, that the difficulty of breathing returned, and the swelling of the legs 
reappeared. In this state he came into the hospital.

Physical Examination. Heart perfectly healthy; left lung perfectly healthy; right lung dull in its lower three-fourths, front and back. Over the dull region there is loss of respiratory murmur, of vocal resonance, and of vocal fremitus. Right side immovable in breathing, all the movement being performed by the left. No egophony. Urine-no albumen; in all respects perfectly healthy.

Now here we evidently have another case of " seneral mechanical" dropsy, only, instead of the heart being the point de depart of the stasis, it is the lung. We have the characteristic distribution of the dropsy ; the presence of the essential symptomdrspnœa, the dropsy varying as the dyspnœa; the kilneys functioning perfectly; the heart healthy; but three-fourths of one lung functionally destroyed -not the seat of respiration, and therefore not the seat of free blood-transit. The diagnosis of this case, in relation to its clinical history and physical signs, is very interesting, and not very clear; but I will not now divert your attention from my main ohject by discussing it. I will merely remark that both this case and the preceding illustrate the fact that you may have extensive œdema of the legs from heart or lung-disease without albuminuria; in other words, that a mechanical source of general dropsy may be sufficient to produce serous transudation from the capillaries of the lower extremities, and yet not be sufficient to produce serous transudation from the capillaries of the kidney. This merely shows that the capillaries of the lower extremities are the most delicate percipients of any source of general stasis of the circulation, and the Malpighian capillaries less so. Formerly, I was not sure that this was the case; but these cases, and other similar ones that $I$ have seen, set the point at rest.

CASE III. William Bush, aged 20, by trade a house-painter, presented himself among the outpatients of this hospital on the 20th of March last, and was at once seen to have colema of the face, which was pallid and puffy. He said he was swollen all over, and showed his legs, which were large and pitting. He stated that his illness commenced some days previously (after having got wet through and suffered his wet clothes and shoes to dry on him) with shivering, pains in the head and loins, swelling of the face, and afterwards feet, legs, and entire body. His urine was examined, and found to be loaded with albumen. He was at once ordered into the hospital.

On admission, we found him in the state described; his face, especially his eyelids, so swollen as to be quite disfigured; the entire cutaneous surface more or less œdematous, the stethoscope leaving a depression wherever it was appliea. The heart and lungs were found to be perfectly healthy, except that the breathing was slightly accelerated; and there was a little dry rhonchus here and there, with short dry cough. He stated that he had been perfectly well up to the commencement of this attack, a few days previously; and that he had never sufjeiel from diffculty of breatling. On examining the urine, we found it so charged with albumen that, on boiling it, the test-tube might be momentarily turned upside-down without anything escaping-so completely was it solidified. It was scanty, and of a specific gravity of 1015 . not bright; and, on putting it aside to stand, an abundant deposit subsided, it aside to stand, an abunda which, on examination by the microscope, was found to be organic, and to consist partly of tube-casts, granular and epithelial, of small and medium size, but chiefly of disintegrated epithelium-cells.

After this patient had been some days in the hospital, he experienced an aggravation of his symptoms from exposure to cold, in consequence of drafts in the wards. We noticed that his face was more puffed; he complained of pains in his loins, and romited; and his urine became charged with blood, in addition to the albumen. From that time forward, however, under appropriate treatment, he has unintcrruptedly improved; the blood soon disappeared; every trace of the dropsy has gone; and all his other symptoms have vanished, except that there is still a trace of albumen in his urine, and may be for some time to come.

Here we have a case manifestly of a different complexion from those which we have just been considering. We have the heart and lungs both perfectly sound, and no dyspnca up to within a few days of his being seen, and even then so slight as not to be a source of any distress. It is manifest, then, that the dropsy is not of the "general mechanical" type, since that arises alone from heart or lung-disease. Moreover, the dropsy is universal, and developed with great rapidity, showing itself not first in the feet and gradually creeping up, but first of all in the face. Moreover, we have such an amount of albumen in the urine as no mechanical source of dropsy would ever produce, and a deposit showing the kidneys to be the seat of primary disease. Two symptoms commonly present in cases of this naturenamely, vomiting and pain in the loins-were absent when the lad came in; but they appeared, as has been narrated, a few days later. That nothing may be wanting, add to all this that the symptoms were preceded by such an exposure to cold and wet as would be sufficient powerfully to check the action of the skin-and the chain of evidence is complete.

It is evident, then, that we have here one of those: cases of what is called "acute dropsy" from surface. cold, always associated with derangement of the kidney, and which I have named "dropsy from. cutaneous asphyxial congestion".

Let us now turn to another case, very similar.

CASE Iv. Louis Tiller, a boy aged 14, was brought: to the hospital the other day, on account of a swelling of his entire body and face, which had suddenly made its appearance a few days previously. He was led into the out-patient room by his mother, his gait feeble, his breathing oppressed, and complaining of headache and pain in his back. But the most striking thing about him was his appearance. His face was bloated, and his eyelids so swollen that his eyes. were very imperfectly opened ; there was a peculiar semitransparent opalescence about his eyelids, and a quaggy fluctuous tremor of them on being gently tapped. The body was so much enlarged that the waistcoat and trousers could not be brought together ; the navel was deeply set; the scrotum was very much swollen; the legs had, from their swelling, lost. the natural shape of a boy's limbs, and they pitted deeply on pressure; the application of anything to any part of the surface of the body left its impression, and the corrugations of the boy's linen were mapped out on many parts of the trunk. In fact, there were all the appearances of universal subcutaneous dropsy. The face and the entire surface of 
the body were blanched. The urine was scanty, and said to be very dark; on having some passed, and examining it, it was at once seen to be darkly smoky from the presence of blood; and, on boiling it, a copious deposit of albumen was thrown down. Heart and lungs healthy; breathing oppressed; no cough; respiratory murmur every where natural: no dyspnoea before the swelling appeared.

You will see at once how like this picture is to the preceding, and how clearly the dropsy is of the same nature. The process of reasoning that would diacnose the one would diagnose the other, and I need not repeat it. The only point of difference in the two cases was the cause. In this latter case, like the other, the dropsy appeared suddenly-the boy went to bed apparently without it, and got up with it; but there was no recognised exposure to wet or cold previously. But inquiry elicited that three weeks before, he had had a rash that was suspected to be scarlatina, and his hands, etc., had peeled. There could be no doubt then that this was a case of "dropsy after scarlatina," and the only difference in respect to the cause was, the particular materies morbi with whose elimination the skin and kidneys were struggling.

CASE v. Within the last few days there has come into the hospital a man exhibiting, if my diagnosis is correct, a form of dropsy which is not a very common one; his history is as follows.

John Bradley, aged 45, has been for some years past a steward on board an Indiaman, and has drunk enormously of spirits. Some short time ago he noticed that he was getting larger in the belly than usual, that he could not button his waistcoat and trousers so well as he formerly could; and this has gone on increasing till now they will not meet by two or three inches; lately his legs have become rapidly œdematous, and are now largely so. On examining the heart there is found no evidence of disease, and in the lungs nothing but slight bronchial sounds apparently quite insufficient to indicate a pulmonary explauation of the dropsy. On physically examining the abdomen it is at once evident that its distension is not due to fluid, as there is not a trace of fluctuation; but we find that the liver is greatly enlarged, occupying the epigastrium, and extending four or five inches below the margins of the right ribs. No albumen in the urine, which is in all respects perfectly healthy.

Now let us trace the process of reasoning by which we arrive at the diagnosis of the nature of the dropsy in this case. In the first place the distributioir of the dropsy is the same as in "general mechanical" - it is dropsy of the lower extremities. But we have not the sources of general mechanical dropsy; the heart and lungs are not the seat of such disease as could generate it ; and we know no otiner source of general mechanical dropsy except disease of the heart or lungs. The kidneys have cleariy nothing to do with it, for they are functioning naturally, and are evidently healthy. But how about the liver? That we find is evidently not healthy. Can that in any way produce the dropsy of the legs? We know that the liver has nothing to do with the circulation of the legs; but that it has to do with another circulation, that of the abdominal viscera, ard that it is on this circulation that discase of the liver tells, and that when it produces dropsy that dropsy is abdominal dropsy, or ascites. But there is one way in which disease of the liver may give rise to œdema of the lower extremities, and that is probably the way in which the dropsy of the legs has been produced in this case. When the liver undergoes great enlargement it becomes a veritable tumour, capable of producing all the effects of a tumour. Immediately behind the posterior border of the liver is the inferior cava, and in a supine position the liver may be said to rest upon it. In health things are so apportioned that no disturbance results; but when the liver is greatly increased in size and weight it presses on the cava, in the supine position, to such a degree as to impede the flow of blood through it, and thus to give rise to the results of pressure on a large venous trunk, one of which results we know to be œdema, corresponding in extent to that segment of the circulation which is tributary to the venous trunk pressed on. Now in this case we have all the conditions for such a production of such a dropsy, and in the absence of any other explanation I cannot but believe that this is the true one. If this is so, this is an example of what I have called "local mechanical" dropsy.

But there is one difficulty in the way of this diagnosis that I must not conceal from you. Any impediment to the return of blood to the heart by the inferior cava produces not only œdema of the lower extremities but enlargement of the superficial veins of the abdomen; now, in this case there is no such enlargement of the superficial abdominal veins. But, at any rate, whether our diagnosis is right or wrong, what I have told you about the case is sufficient to illustrate the principles upon which the diagnosis of local mechanical dropsy is based.

Case vr. Joseph Slater, aged 61 , an agricultural labourer, was admitted into the hospital for ascites and swelling of the feet and legs. Of temperate habits, and has always had good health. He first noticed the swelling of his belly early in February; it gradually increased, and he came to the hospital. $\mathrm{He}$ had had pains in the back, but none in the belly; had lost flesh and strength; the bowels had acted regularly; the urine was high-coloured, turbid, and scanty.

On examining the patient we found him emaciated and sallow, and the abdominal cavity enormously distended with fluid-the circumference of the abdomen forty-five inches; the thrill on percussion highly dereloped in consequence of the tension with which the thin parietes were stretched over the fluid; the superficial abdominal veins enlarged. There was one appearance which was rather peculiar ; the umbilical opening was very much enlarged, about an inch in diameter, and through it projected a sort of hemisphere of peritoneum, driven forward by the fluid beneath it, and covered by the thinned integument above it, constituting a sort of serous hernia; it was about as big as half a large walnut. The characteristic thrill could be elicited in this little cavity, and when the light shone through it it had the semitransparency of a hydrocele. No outline of the liver could be made out. The legs were but little swollen, chiefly about the ankles. The motions were pale; the urine evidently containing bile; no albumen. Heart and lungs healthy.

In this case you see the features of the dropsy were very well marked, and were of an entirely different character from those of the previous cases; 
and up to a certain point the diagnosis was perfectly clear. The urine asserted the kidneys to be healthy, and the stethoscope asserted the heart and lungs to be healthy. There seemed nothing, then, left but the liver. 'That this was the organ at fault was further shown by the sallow skin, the pale motions, and the bilious urine. Moreover, the particular form of the dropsy-ascites-was that which directly points to the liver. But there was just one point that raised a doubt as to whether the liver itself was the seat of the organic change causing the dropsy ; it was quite clear that that cause was something impeding the portal circulation and hindering the escape of bile ; but it might be all that, and yet not the liver. I have mentioned that the feet were swollen and the superficial veins of the abdomen enlarged; it seemed, then, that the disease, whatever it was, was something exercising pressure on the inferior cava; if this was the liver the organ ought to be enlarged, so as to constitute a tumour and an incumbent weight on that vessel. But no trace of enlargement of the liver could be found; and $I$ may mention that afterwards, when the man was tapped and the dropsy became, therefore, less a source of obscuration, no enlargement of the liver could be detected. It seemed, therefore, to be more probable that the source of the dropsy was some disease (it might be cancer) not involving the whole liver texturally, but either foreign to the liver or growing from the liver, and so placed as to press on the portal vein, hepatic duct, and inferior cava.

The man died (I purposely avoid now all further detail of the case), and the post mortem examination shewed that this refinement of diagnosis was wrong, that the cause of the dropsy was a textural degeneration of the liver, not attended with an increase of its bulk, but with a great diminution of it, the liver weighing only a pound and a haif. Another case which was in the hospital at the time, and which I will now relate, happily illustrated, a short time subsequently, where the fallacy of our reasoning was.

CASE vir. John Holland, aged 40, was admitted into Charing Cross Hospital June 3rd, with an enormously distended belly and odematous legs. He had led a very wild and irregular life, and had drunk an inmensity of spirits. His symptoms were of many months standing, and into them romiting and jaundice had largely entered. $\mathrm{He}$ was emaciated, pallid, and sallow, the sallowness sometimes rising to jaundice. Heart and lungs healthy ; urine containing no albumen. There was the same hernial protrusion of the peritoneal sac at the umbilicus as in Slater's case, only to a much greater extent-as big as a hen's egrg; and the legs were much more œdematous, as much so as one often sees in an advanced stage of heart-disease. There was this difference, too-that in spite of the drummy tension of the abdomen with fluid, and the dulness which this produced, we thought we could detect great enlargement of the liver. Afterwards, when the man was tapped, and the serum (twenty-four pints) was drawn away, this became very conspicuous, and the lax parietes could be thrust beneath the margin of the liver, and fairly under the organ, thus bringing its edge into view, which was five or six inches below its natural seat.

There seemed, then, no difficulty in explaining the double dropsy in this case-the ascites was owing to a textural disease of the liver, probably cirrhotic, impeding the transit of blood through it, while the œdema of the legs was due to the pressure of the: enlarged organ back on the inferior cava. But mark what followed. When this man was tapped his legs. and thighs were enormously œdematous, more so than they had been at all; within eight and forty hours afterwards not a trace of cederina was to be seen.

From this sudden disappearance of the cedema of the legs after the tapping, we learned two things :-

]. That the pressure of the enlarged liver on the inferior cava was not the cause of the œdema of the legs ; for the liver was just as large after the tapping, but the œdema was gone.

2. That the pressure of the incumbent fluid on the great inferior systemic vessels was the cause of it, and, therefore, might be the cause of it in Slater's, or any other case. This proof that ascites is of itself sufficient to generate so great an amount of œdema of the lower extremities is a most important clinical fact, as it removes the necessity for any other explanation of dropsy of the legs in any case in which ascites is present. Had I known this when I was diagnosing Slater's case I should have been content with the hypothesis of simple liver disease, as a sufficient interpretation of it.

Gentlemen, I must here conclude my lecture. There are yet two forms of dropsy whose diagnosis I have not illustrated; for the good and sufficient reason that I have no cases wherewith to illustrate them ; one, the primary dropsy of serous cavities; the other, the dropsy of watery blood. I must illustrate these on some future occasion.

How Inoculation came about. At a time when small-pox was as destructive as the plague itself, Lady Mary Wortley Montagu, happening to be at Adrianople, was struck with the fact that the Turks were in the habit of making terms with the disease by receiving it into their system by way of the slin, instead of by the lungs, as in the natural mode of infection. Possibly, the lively nature of the lady's letters had more to do with the sensation this new practice created in England than the magnitude of the truth she made known, and to this day we believe that the public have some idea that it was a discovery made by her ladyship, and which she had the boldness to put in practice upon her own son. Yet no fact is more certain than that throughout Asia the practice of inoculation had obtained for ages; and that the Chinese-the inevitabie nation to which we have always to go back for the birth of any great discoverysystematically employed inoculation as early as the sixth century. Yet, strange to say, in Asia this precious knowledge came to a dead standstill, and had it not been for the lively English lady inoculation might not bave been introduced into England for another half-century, and possibly vaccination would even now be in the womb of time. 'That inoculation was a grand step towards the practice of vaccination there can be little doubt, although science did not at the time appreciate the fact. It taught us that the disease received into the circulation by the skin was infinitely less dangerous than the disease "caught" by inhalation through the lungs, a circum. stance which mediciue cannot explain to this day. The deaths from small-pox during some of the severe epi. demics of the last century were not less than a third of those attacked, but the improved practice of inoculation reduced these deaths to one in two hundred. (Once a Week.) 